\title{
The advancement of utilizing geospatial information via GSI Maps
}

\author{
Hirosato Mogi ${ }^{\text {a, }}$ * \\ ${ }^{a}$ Geospatial Information Authority of Japan, Ibaraki, Japan, mogi-h96sq@mlit.go.jp \\ * Corresponding author
}

Keywords: Open source software, Open data, Web map

\section{Abstract:}

The Geospatial Information Authority of Japan (GSI) is the national organization that has jurisdiction over the Survey Act, and develops geospatial information. We are also promoting utilization of them based on the Basic Act on the Advancement of Utilizing Geospatial Information. In addition, the Japanese government is promoting the Open Data initiative, in which the government widely discloses public data in machine-readable formats and allows secondary use of them. In accordance with these laws and initiative, we aim to realize a society where geospatial information can be widely and highly utilized by the disclosure of geospatial information. In order to achieve our mission, we are working on providing map data using "GSI Maps" (https://maps.gsi.go.jp/) which is a web map developed by using open source software (OSS) as the basis (Figure 1a). In this paper, we introduce "Three Open Policies" to promote utilizing geospatial information provided via GSI Maps.

We provide various types of tiled geospatial data including topographic maps, aerial photographs, thematic maps and disaster information. These data are called "GSI Tiles" (Figure 1b). GSI Maps works as a showcase of GSI Tiles with basic functions (for example, measurement of distance and area). GSI Maps and GSI Tiles are available via the web. That is important to achieve our mission because whoever has a device with a web browser and Internet connection can access them.

Web Server of GSI

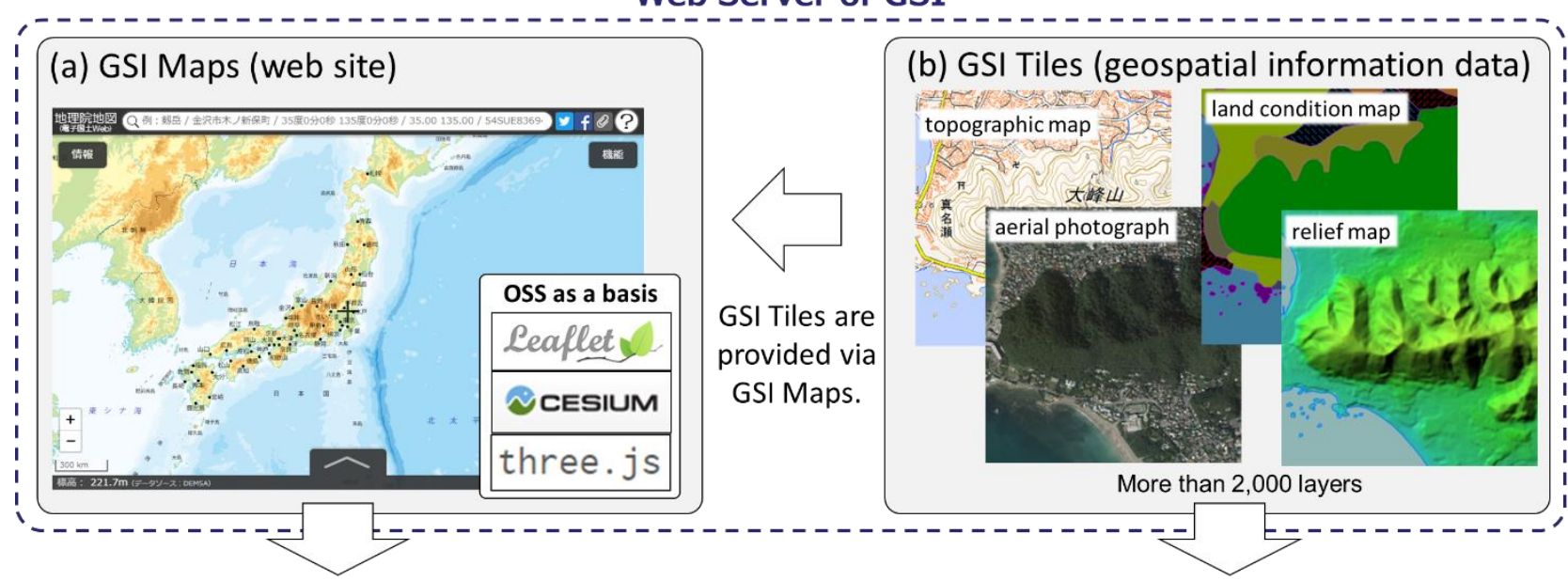

(d) The source codes of GSI Maps are provided as OSS on GitHub.

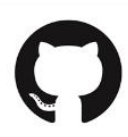

(c) GSI Tiles as Open Data are utilized via various applications.

\begin{tabular}{|c|c|}
\hline Web Application & GIS Software \\
\hline Native Application & etc. \\
\hline
\end{tabular}

Figure 1. Schematic illustration of GSI Maps and GSI Tiles.

Users can also utilize GSI Tiles in applications other than GSI Maps. The direct access to GSI Tiles, not via GSI Maps, is also encouraged (Figure 1c). We aim to promote the utilization of GSI Tiles with Three Open Policies including the Open Data Policy, the Open Source Policy and the Open Innovation Policy.

As the Open Data Policy, we open GSI Tiles to the public under the Geospatial Information Authority of Japan Website Terms of Use (Version 2.0) (http://www.gsi.go.jp/ENGLISH/page_e30286.html), which is compatible with Creative Commons Attribution 4.0 International Public License (https://creativecommons.org/licenses/by/4.0/legalcode). The 
format of GSI Tiles is slippy map tilenames (https://wiki.openstreetmap.org/wiki/Slippy_map_tilenames) supported by various platforms including a lot of OSS. Therefore we can flexibly adopt the base software of GSI Maps without changing the data format. Actually, in 2015, we changed the base OSS to another one, "Leaflet" (https://leafletjs.com/). We also developed "GSI Maps 3D" using "three.js" (https://threejs.org/) that enables users to view in 3D and download the data for 3D printers. In addition, we built a seamless 3D web map, "GSI Globe” using "Cesium” (https://cesiumjs.org/).

As the Open Source Policy, OSS are utilized to build GSI Maps. We have provided a web map since 2003. Although, at first, we developed it from scratch, in 2011, "OpenLayers" (http://openlayers.org/) was adopted as the basis. The first reason to adopt OSS is to reduce the development cost. The second reason is to prevent vendor lock-in. Since the disclosure of derivative works of OSS is permitted, the source codes of GSI Maps are also released as OSS on GitHub (https://github.com/gsi-cyberjapan) (Figure 1d). These codes are helpful to develop websites with GSI Tiles other than GSI Maps (e.g. road information websites, and mountaineering websites). Actually, some local governments have built their own web maps based on GSI Maps sources. There are some reports that in certain local governments, the maintenance cost have been reduced compared with the past by constructing a web map site with reference to GSI Maps.

As the Open Innovation Policy, we organize the "GSI Maps Partner Network", which is composed of GSI and partners who utilize GSI Tiles, including 100 contracted developers and 69 tool providers (as of November 2018). In this network, we pursue innovative utilization of geospatial information in various fields. We hold conferences twice a year to share the information on recent efforts for GSI Maps and GSI Tiles by GSI, and the utilization of GSI Tiles by partners.

As a result of such efforts based on Three Open Policies, the request number of GSI Tiles has been increasing year by year. Figure 2 shows the temporal changes of the request number to GSI Tiles from April 2014 to October 2018. We note that the requests from web pages and applications other than GSI Maps (square symbols ( $\square$ ) in Figure 2) account for about $80 \%$ of all requests. To activate the innovative use of geospatial information in the various field, we will continue to provide GSI Maps and GSI Tiles, as well as promote the sharing of data use cases, in accordance with our Three Open Policies.

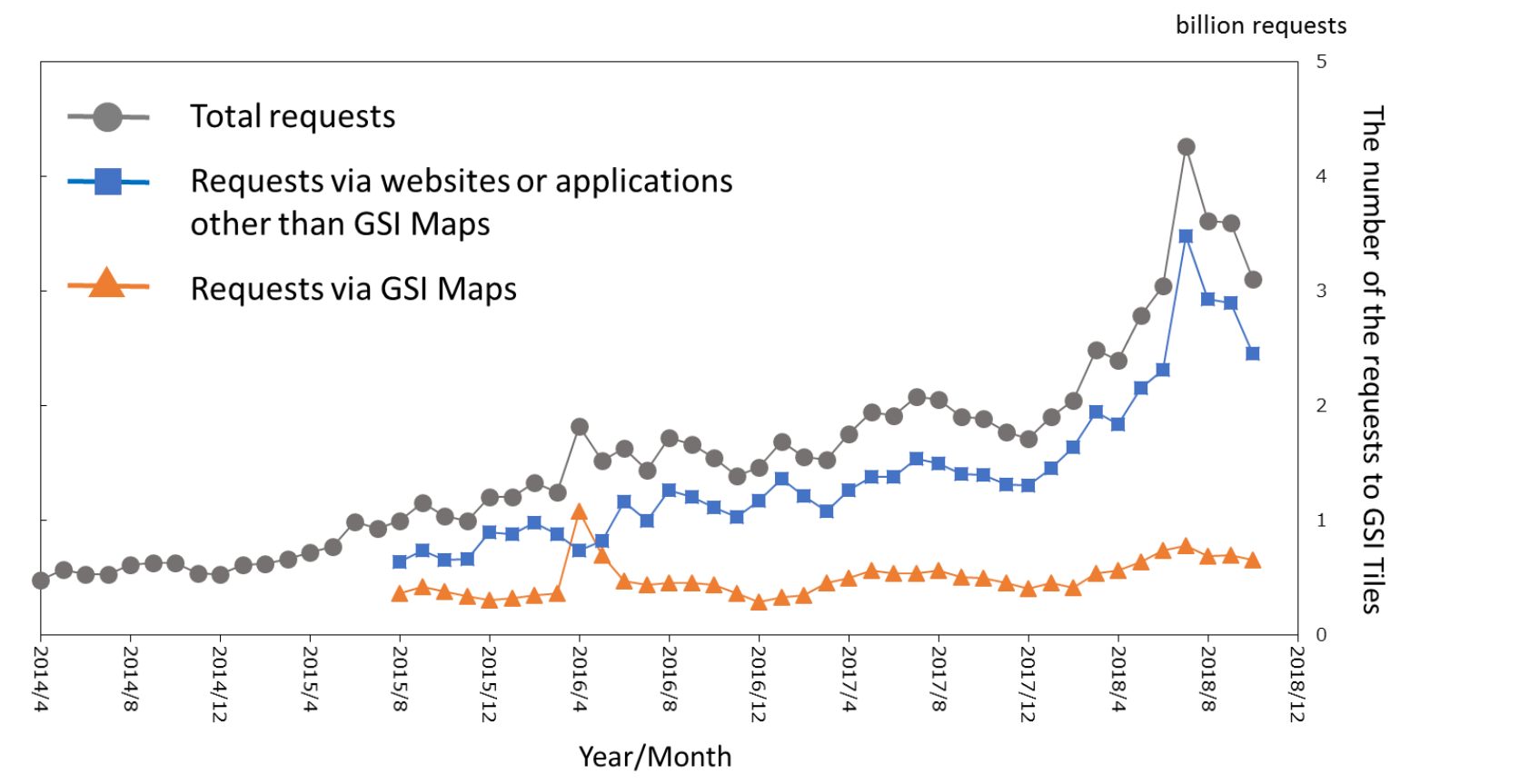

Figure 2. The temporal changes of the request number to GSI Tiles from April 2014 to October 2018. Symbols show the total request number $(\bullet)$, the request number via GSI Maps $(\boldsymbol{\Delta})$, and the request number via websites or applications other than GSI Maps (ם). 